\title{
Conhecimento e comportamento de um grupo de idosos frente às infecções sexualmente transmissíveis
}

\author{
Knowledge of a group of elderly people about sexually transmitted infections and their \\ behavior towards these diseases \\ Conocimiento y comportamiento de un grupo de ancianos contra las infecciones de \\ transmisión sexual
}

Samantha Vieira Alves Amaral ${ }^{1 *}$, Renata Lacerda Prata Rocha ${ }^{1}$, Vinicius Siqueira Seabra Junqueira ${ }^{1}$, Lorraine Dayanne Moreira Martins ${ }^{1}$, Hemilly Mendonça Souza ${ }^{1}$, Pâmela Malheiro Oliveira², Andréa Tayse de Lima Gomes², Gislene Pace de Souza Santos².

\section{RESUMO}

Objetivo: Compreender sobre o comportamento e o conhecimento de um grupo de idosos frente às Infecções Sexualmente Transmissíveis (IST's). Métodos: Foi realizada uma pesquisa de campo, de delineamento quantitativo, com 59 idosos participantes de um grupo de convivência em um município de Minas Gerais (MG), no período de julho a outubro de 2018. Resultados: O estudo evidenciou que os idosos possuem certo conhecimento acerca das IST'S e de suas formas de transmissão. Dentre as IST'S a mais conhecida pelos entrevistados é a HIV/AIDS (64\%). Porém a maioria adota comportamento de risco ao não usar o preservativo nas relações sexuais $(76,3 \%)$, e $81,4 \%$ deles não se consideram vulneráveis para adquirir uma IST, justificando tal fato por terem parceiro fixo. Um achado positivo é a ciência da existência da vacina contra a hepatite B $(50,8 \%)$, identificando-a como uma doença imunoprevenível, disponível inclusive para sua faixa etária. Conclusão: $O$ estudo denota a necessidade de práticas mais assertivas voltadas a esse público de maneira a sensibilizá-los quanto a importância de se proteger nas relações sexuais.

Palavras-chave: Idoso, Doenças sexualmente transmissíveis, Enfermagem.

\begin{abstract}
Objective: This work attempts to analyze and understand the knowledge level of Sexually Transmitted Infections (STIs) in a group of elderly people and their behavior regarding such diseases. Methods: A field research with quantitative delineation was performed with 59 seniors, participants in a community group in a municipality in Minas Gerais (MG), from July to October 2018. Results: The study has revealed that, although the aged know STIs and their ways of transmission. Among the STIs the most known by the interviewees is HIV / AIDS (64\%). Most adopt risky behavior when not using condoms during sexual intercourse (76.3\%), and $81.4 \%$ of them do not consider themselves vulnerable to acquire an STI, justifying this fact because they have a steady partner. A positive finding is the knowledge of the existence of the vaccine against hepatitis $B(50,8 \%)$, identifying it as an immunopreventable disease, available even for its age group. Conclusion: The study shows the need for affirmative practices better suited to that public, in order to raise its awareness of the importance of protecting themselves in sexual relations.
\end{abstract}

Keywords: Aged, Sexual transmitted diseases, Nursing.

\section{RESUMEN}

Objetivo: Comprender el comportamiento y el conocimiento de un grupo ancianos frente a las infecciones de transmisión sexual (ITS). Métodos: Se realizó una investigación de campo, con un diseño cuantitativo, con

${ }_{1}$ Centro Universitário UNA. Belo Horizonte - MG. *E-mail: samanthavalves@gmail.com

2 Universidade Federal de Minas Gerais (UFMG). Belo Horizonte - MG. 
59 ancianos de un grupo comunitario en un municipio de Minas Gerais (MG), de julio a octubre de 2018. Resultados: El estudio mostró que los ancianos tienen conocimiento sobre las ITS y sus formas de transmisión. Entre las ITS, la más conocida por los entrevistados es el VIH / SIDA (64\%). La mayoría adopta conductas de riesgo por no usar condones durante las relaciones sexuales $(76.3 \%)$, y el $81.4 \%$ de ellos no se consideran vulnerables a contraer una ITS, lo que justice este hecho porque tienen una pareja estable. Un hallazgo positive es el conocimiento de la existencia de la vacuna contra la hepatitis B (50,8\%), identificándola como una enfermedad inmunoprevenible, disponible incluso para su grupo de edad. Conclusión: El estudio muestra la necesidad de prácticas más asertivas dirigidas a esta populación para que sean conscientes de la importancia de protegerse en las relaciones sexuales.

Palabras clave: Ancianos, Enfermedades sexualmente transmisibles, Enfermería.

\section{INTRODUÇÃO}

O mundo está envelhecendo, e esta realidade decorre dos avanços contínuos na tecnologia tanto na área médica, quanto na indústria farmacêutica, bem como nos sistemas públicos de saúde e nas condições socioeconômicas da população (NETO JD, et al., 2015). No Brasil, o envelhecimento populacional é marcado pela transição demográfica decorrente da diminuição das taxas de natalidade e mortalidade, que culminaram no aumento da expectativa de vida. Em 1920, a expectativa de vida era de apenas 35,2 anos e os idosos representavam apenas $4 \%$ da população total do país. Atualmente a expectativa de vida é de 75,8 anos e correspondem a 14\% da população geral (MIRANDA GMD, et al., 2016; MASCHIO MBM, et al., 2011; IBGE, 2018).

Contudo o aumento da expectativa de vida veio acompanhado do aumento de algumas doenças entre os idosos, entre elas as infecções sexualmente transmissíveis (IST's). Conforme dados do Ministério da Saúde, a Síndrome da Imunodeficiência Humana Adquirida (AIDS) na faixa etária de 60 anos ou mais registrou um aumento dos casos passando de 7,3 casos por 100.000 habitantes em 2005 para 9,3 casos em 2016 (MS, 2017a).

Em relação à sífilis adquirida, na faixa etária de 60 anos ou mais, verificou-se a notificação de 703 casos em 2010; e 8.311 casos, no ano de 2017 (MS, 2017c). Já a hepatite B, na faixa etária de 60 anos ou mais, teve em 2003 uma taxa de detecção de 2,9 em 100.000 habitantes, aumentando para 7,8 em 2016 (MS, 2017b). Esses números revelam uma realidade preocupante: os idosos estão vulneráveis as IST's e precisam de orientações adequadas com o propósito de evitá-las (MASCHIO MBM, et al., 2011).

A probabilidade de um idoso ser infectado por alguma IST parece invisível aos olhos da sociedade. Para muitos o sexo é algo a ser vivido somente durante a juventude, e logo, esse assunto na terceira idade se torna cercado de tabus e preconceitos, que advém de diversos setores da sociedade, inclusive de profissionais de saúde (ANDRADE JAA, et al., 2017). Até mesmo entre os próprios idosos verifica-se a falta da cultura de prevenção com a adoção muitas vezes de comportamentos de risco, tais como práticas sexuais sem o uso de preservativos.

Para os idosos, principalmente do sexo masculino o uso do preservativo ainda é um tabu; e para as mulheres idosas, uma prática desnecessária, por não estarem mais em período fértil. Além disso, a falta de políticas públicas voltadas a este grupo populacional, culmina no aumento de atitudes de risco e na incidência de IST's na população acima de 60 anos (ANDRADE JAA, et al., 2017; BURIGO GF, et al., 2015; SOUZA LPS, et al., 2013; MASCHIO MBM, et al., 2011).

No entanto, a sexualidade entre a população da terceira idade está rompendo paradigmas, se despindo de suposições e estereótipos antigos. Muitos idosos, inclusive acima dos 80 anos, ainda se encontram sexualmente ativos em decorrência da melhoria de seu estilo de vida, realização de tratamentos hormonais e acesso a medicações utilizadas para potencializar o desempenho sexual (ANDRADE JAA, et al., 2017; NETO JD, et al., 2015; SOUZA LPS, et al., 2013).

Nesse contexto, é fundamental que a equipe multidisciplinar se aprimore para assistir essa população, atendendo de forma integral, suprindo suas necessidades e anseios. Em especial, o enfermeiro, deve assumir 
o seu papel como educador em saúde, com práticas que envolvam a minimização dos fatores de risco, empoderamento por meio do acesso à informação, e alinhamento de adoção de hábitos de vida saudáveis que motivem um envelhecimento sadio, permitindo o redescobrimento de novas experiências tais como 0 sexo seguro (TOLDRÁ RC, et al., 2014; NETO JD, et al., 2015).

Portanto, o envelhecimento é uma realidade e necessita ser discutido no contexto da sexualidade e do aumento da incidência de IST's em idosos. Nesse sentido esse estudo é relevante, pois os resultados alcançados podem revelar lacunas do conhecimento que ainda precisam ser trabalhadas. Pode-se ainda estimular a reflexão do papel da equipe multidisciplinar no âmbito da educação em saúde, bem como do estímulo das práticas de autocuidado e acesso à informação. É essencial motivar o idoso a tornar-se protagonista do seu autocuidado, vivenciando diversas experiências, incluindo o sexo, com mais segurança. Embora tal problemática esteja em destaque, ainda há poucas investigações envolvendo essa questão, especialmente no Brasil.

Dessa forma, o presente estudo tem como objetivo geral compreender o comportamento e o conhecimento de um grupo de idosos frente às Infecções sexualmente transmissíveis, e como objetivos específicos: caracterizar o perfil socioeconômico do grupo de idosos; identificar o conhecimento dos idosos sobre as Infecções sexualmente transmissíveis e seu comportamento sexual.

\section{MÉTODOS}

Trata-se de um estudo de campo exploratório-descritivo, com abordagem quantitativa, realizado entre julho a outubro de 2018 com idosos participantes de um grupo de convivência em um município de Minas Gerais (MG). Fazem parte desse grupo, cerca de 120 idosos.

Foram incluídos na pesquisa, todos os idosos lúcidos e orientados, que concordaram em participar voluntariamente e que apresentaram capacidade cognitiva para responder o questionário apresentado. Foram excluídos do estudo, os idosos que não estavam presentes no momento da coleta de dados e aqueles que não sabiam ler. Esse último critério foi aplicado, tendo em vista que, por se tratar de uma temática relacionada à sexualidade do idoso, supôs-se que poderia surgir um constrangimento caso os pesquisadores aplicassem diretamente o questionário face a face.

A amostra dessa pesquisa foi do tipo não probabilística determinada por conveniência. Assim, de um universo de 120 idosos, 65 foram elegíveis para participar da pesquisa, e constituírem a amostra. No entanto, a amostra final constitui-se de 59 idosos, pois após a coleta de dados, 6 questionários foram excluídos por não estarem preenchidos na grande maioria das questões.

A coleta de dados se deu através da aplicação de um questionário objetivo, composto por 27 questões. $O$ instrumento foi elaborado a partir do referencial teórico do Ministério da Saúde (MS, 2014; MS, 2017c). O instrumento constitui-se de três partes: Parte I: características sociodemográficas; Parte II: conhecimentos sobre as IST's, e a Parte III: comportamento sexual. Para verificação do conhecimento dos participantes sobre as IST's, foram elencadas as seguintes patologias: HIV (Vírus da Imunodeficiência Humana)/AIDS (Síndrome da imunodeficiência adquirida), sífilis, HPV (Papilomavírus Humano) e hepatite B. Nos questionários a palavra "AIDS" (foi utilizada como sinônimo de HIV para melhor entendimento dos questionamentos, já que tal termo é mais utilizado no senso comum, apesar de designarem terminologias diferentes.

$\mathrm{Na}$ análise dos dados realizou-se a Estatística Descritiva Simples. Para verificar as variáveis, em especial o conhecimento e comportamento dos idosos, utilizou-se o programa estatístico Epi info versão 3.4.5, para Windows de domínio público. Ao final do tratamento dos dados, foram construídas tabelas através do software Excel 2016, cuja consolidação serviu para realização de associações embasadas pela literatura científica sobre a temática em questão.

A pesquisa foi aprovada pelo Comitê de Ética em Pesquisa do Centro Universitário UNA, conforme CCAE no 94986618.0.0000.5098. Durante toda a sua realização os participantes foram orientados quanto aos objetivos da pesquisa, sobre a garantia do anonimato e do direito de desistência em qualquer fase do estudo. Além disso, foram esclarecidos com relação ao aceite voluntário do convite, e da necessidade de assinatura do Termo de Consentimento Livre e Esclarecido para anuência de sua participação conforme preconiza a Resolução 510/2016 (MS, 2016). 


\section{RESULTADOS}

Dentre as características socioeconômicas dos idosos participantes do estudo verifica-se um predomínio do sexo feminino ( $n=56,94,9 \%)$ com idade entre 70 e 79 anos $(n=30,50,8 \%)$, estado civil casado ( $n=28$, $47,5 \%)$, aposentados ( $n=39,66,1 \%)$, e religião católica $(n=39,66,1 \%)$. Em relação a utilização dos serviços de saúde, apenas $22 \%(n=13)$ relatou possuir alguma forma de convênio. Quanto ao grau de escolaridade, $71,2 \%(n=42)$ afirma ter de 1 a 4 anos de estudo. E nos aspectos econômicos, 76,3\% ( $n=45)$ afirma um ganho mensal entre 1 a 5 salários mínimos (Tabela 1).

Tabela 1 - Caracterização dos idosos segundo variáveis socioeconômicas, $n=59$. Belo Horizonte, Minas Gerais, 2018.

\begin{tabular}{|c|c|c|}
\hline Variáveis & $\mathbf{N}$ & $\%$ \\
\hline \multicolumn{3}{|l|}{ Sexo } \\
\hline Feminino & 56 & 94,9 \\
\hline Masculino & 03 & 05,1 \\
\hline \multicolumn{3}{|l|}{ Idade } \\
\hline $60-70$ anos & 22 & 37,3 \\
\hline $70-80$ anos & 30 & 50,8 \\
\hline $80+90$ anos & 05 & 08,5 \\
\hline$\geq 90$ anos & 02 & 03,4 \\
\hline \multicolumn{3}{|l|}{ Estado civil } \\
\hline Solteiro & 03 & 05,1 \\
\hline Casado & 28 & 47,5 \\
\hline Viúvo & 24 & 40,7 \\
\hline Divorciado & 04 & 06,7 \\
\hline \multicolumn{3}{|l|}{ Ocupação } \\
\hline Aposentado & 39 & 66,1 \\
\hline Ativo no mercado de trabalho & 06 & 10,2 \\
\hline Sem resposta & 14 & 23,7 \\
\hline \multicolumn{3}{|l|}{ Convênio médico } \\
\hline Sim & 13 & 22 \\
\hline Não & 46 & 78 \\
\hline \multicolumn{3}{|l|}{ Religião } \\
\hline Evangélico & 07 & 11,9 \\
\hline Católico & 39 & 66,1 \\
\hline Outras & 05 & 08,4 \\
\hline Não respondeu & 08 & 13,6 \\
\hline \multicolumn{3}{|l|}{ Escolaridade } \\
\hline Analfabeto & 00 & 0 \\
\hline 1 a 4 anos de estudo & 42 & 71,2 \\
\hline 5 a 8 anos de estudo & 07 & 11,9 \\
\hline 9 a 11 anos de estudo & 06 & 10,2 \\
\hline Acima de 12 anos & 04 & 06,8 \\
\hline \multicolumn{3}{|l|}{ Renda Individual } \\
\hline 1 a 5 salários mínimos & 45 & 76,3 \\
\hline 6 a 10 salários mínimos & 04 & 06,8 \\
\hline Acima de 10 salários mínimos & 01 & 01,7 \\
\hline Sem renda & 06 & 10,2 \\
\hline Não respondeu & 03 & 05,1 \\
\hline Total & 59 & 100 \\
\hline
\end{tabular}

Fonte: Amaral SVA, et al., 2020. 
Relacionado ao conhecimento dos participantes sobre as formas de transmissão e prevenção das IST'S e os aspectos fisiopatológicos relacionados às doenças selecionadas para o estudo. Observa-se que com relação as patologias que conheciam, $64,4 \%(n=38)$ referiu a doença HIV/AIDS e somente $27,1 \%(n=16$, afirmou conhecer todas as doenças questionadas. Sobre a importância do uso métodos de prevenção durante a relação sexual, a maioria respondeu corretamente sobre o uso do preservativo para prevenir IST e evitar gravidez ( $n=31,52,5 \%$ ) (Tabela 2).

Quanto à vulnerabilidade relacionada às pessoas que podem adquirir uma IST 78\% ( $n=46)$ acredita que qualquer pessoa pode adquiri-las. Quando questionados sobre quais patologias eles achavam que não tinha cura, uma parcela significativa indicou a "doença" HIV/AIDS ( $n=25,42,4 \%)$. Para a grande parte dos idosos participantes do estudo, há vacina para prevenir a hepatite $B(n=30,50,8)$. No tocante à transmissão das IST's, a grande maioria dos idosos respondeu corretamente que elas podem ser transmitidas durante a relação sexual desprotegida $(n=45,76,3 \%)$. 
Revista Eletrônica Acervo Saúde / Electronic Journal Collection Health | ISSN 2178-2091

Tabela 2 - Conhecimento dos idosos sobre as Infecções Sexualmente Transmissíveis, n= 59. Belo Horizonte, Minas Gerais, 2018.

\begin{tabular}{|c|c|c|}
\hline Variáveis & $\mathbf{N}$ & $\%$ \\
\hline \multicolumn{3}{|l|}{ Quais dessas IST conhece } \\
\hline HIV/AIDS & 38 & 64,4 \\
\hline HPV & 21 & 35,5 \\
\hline Sífilis & 21 & 35,5 \\
\hline Hepatite B & 24 & 40,6 \\
\hline Todas & 16 & 27,1 \\
\hline Sem resposta & 08 & 13,6 \\
\hline \multicolumn{3}{|l|}{ Importância do uso de preservativo } \\
\hline Somente prevenir IST & 19 & 32,2 \\
\hline Somente prevenir gravidez & 02 & 03,4 \\
\hline Prevenir IST e gravidez & 31 & 52,5 \\
\hline Não é necessário usar & 04 & 06,8 \\
\hline Sem resposta & 03 & 05,1 \\
\hline \multicolumn{3}{|l|}{ Quem pode adquirir uma IST } \\
\hline Somente profissionais do sexo & 03 & 05,1 \\
\hline Somente usuários de drogas & 02 & 03,4 \\
\hline Somente homossexuais & 05 & 08,4 \\
\hline Qualquer pessoa & 46 & 78,0 \\
\hline Sem resposta & 03 & 05,1 \\
\hline \multicolumn{3}{|l|}{ Qual patologia não tem cura } \\
\hline HIV/AIDS & 25 & 42,4 \\
\hline HPV & 09 & 15,3 \\
\hline Hepatite B & 06 & 10,1 \\
\hline Sífilis & 03 & 05,1 \\
\hline Sem resposta & 16 & 27,1 \\
\hline \multicolumn{3}{|l|}{ IST que tem vacina para o idoso } \\
\hline HIV/AIDS & 07 & 11,8 \\
\hline Hepatite B & 30 & 50,8 \\
\hline Sífilis & 06 & 10,1 \\
\hline HPV & 15 & 25,4 \\
\hline Sem resposta & 13 & 22,0 \\
\hline
\end{tabular}

REAS/EJCH | Vol.12(9) | e3891 | DOI: https://doi.org/10.25248/reas.e3891.2020 Página 6 de 12 
Revista Eletrônica Acervo Saúde / Electronic Journal Collection Health | ISSN 2178-2091

\begin{tabular}{|c|c|c|}
\hline Variáveis & $\mathbf{N}$ & $\%$ \\
\hline \multicolumn{3}{|l|}{ Sinal/sintoma do HPV } \\
\hline Verrugas na região genital & 29 & 49,2 \\
\hline Febre & 08 & 13,6 \\
\hline Tosse & 07 & 11,8 \\
\hline Dor muscular & 04 & 06,8 \\
\hline Sem resposta & 11 & 18.6 \\
\hline \multicolumn{3}{|l|}{ Transmissão das IST'S } \\
\hline Durante a relação sexual desprotegida & 45 & 76,3 \\
\hline Pela saliva através do beijo & 08 & 13,6 \\
\hline Pelo compartilhamento de talheres e copos & 02 & 03,4 \\
\hline Pelo abraço & 00 & 00,0 \\
\hline Sem resposta & 04 & 06,7 \\
\hline \multicolumn{3}{|l|}{ Sinal/sintoma da Sífilis na fase inicial } \\
\hline Mal estar & 07 & 11,9 \\
\hline Dor de cabeça & 05 & 08,5 \\
\hline Dor durante a relação sexual & 12 & 20,3 \\
\hline Uma ferida indolor e endurecida na região genital & 25 & 42,4 \\
\hline Sem resposta & 10 & 16,9 \\
\hline \multicolumn{3}{|l|}{ Sinal/sintoma da hepatite B } \\
\hline Icterícia (amarelão), dor abdominal e urina escura & 43 & 72,9 \\
\hline Dor atrás dos olhos e visão escurecida & 03 & 05,1 \\
\hline Dor no peito e falta de ar & 03 & 05,1 \\
\hline Dor ao urinar & 03 & 05,1 \\
\hline Sem resposta & 07 & 11,8 \\
\hline \multicolumn{3}{|l|}{ Sinal/sintoma do HIV/AIDS na fase tardia } \\
\hline Aparecimento de doenças oportunistas & 26 & 44,1 \\
\hline Deixa de ser transmissível pela relação sexual & 03 & 05,1 \\
\hline Causa dor ao urinar & 05 & 08,5 \\
\hline Causa desconforto na relação sexual & 06 & 10,2 \\
\hline Sem resposta & 19 & 32,1 \\
\hline Total & 59 & 100 \\
\hline
\end{tabular}

Fonte: Amaral SVA, et al., 2020. 
O comportamento sexual é retratado nos últimos seis meses, a maioria afirmou que não teve relação sexual $(n=42,71,2 \%)$. Quando indagados sobre o uso de algum método de prevenção $45,8 \%(n=27)$ afirma que quando tem atividade sexual, "às vezes" fazem o uso preservativo, enquanto que $23,7 \%(n=14)$ referiu "nunca" utilizar, desses $39 \%$ ( $n=16)$ justificou que não utiliza por possuir parceiro fixo, ou por não achar necessário ( $n=13,31,7 \%)$. A maioria não se considera vulnerável para adquirir uma IST $(n=48,81,4)$ (Tabela 3). Ao serem questionados sobre como se sentem em relação a falar sobre sexualidade, $59,3 \%$ afirmou sentirse à vontade para conversar sobre esse tema $(n=35,59,3 \%)$. Porém, dos $40,7 \%(n=24)$ que não se sentem à vontade, $45,7 \%(n=16)$ deles justificam por acharem que idoso não tem relação sexual e $33,3 \%(n=8)$ por vergonha.

Tabela 3 - Comportamento sexual dos idosos participantes do estudo, $n=59$. Belo Horizonte, Minas Gerais, 2018.

\begin{tabular}{|c|c|c|}
\hline Variáveis & $\mathbf{N}$ & $\%$ \\
\hline \multicolumn{3}{|l|}{ Teve relação sexual nos últimos 6 meses } \\
\hline Sim & 17 & 28,8 \\
\hline Não & 42 & 71,2 \\
\hline \multicolumn{3}{|l|}{ Usa preservativo durante as relações sexuais } \\
\hline Sempre & 09 & 15,3 \\
\hline Ás vezes usa & 27 & 45,8 \\
\hline Nunca usa & 14 & 23,7 \\
\hline Sem resposta & 09 & 15,3 \\
\hline \multicolumn{3}{|l|}{ Se nunca usa ou as vezes usa, por quê? } \\
\hline Tem medo de perder a ereção & 0 & 00,0 \\
\hline Tem parceiro fixo & 16 & 39,0 \\
\hline Não tem risco de engravidar & 05 & 12,2 \\
\hline Não acha necessário & 13 & 31,7 \\
\hline Sem resposta & 07 & 17,1 \\
\hline $\begin{array}{ll}\text { Total } \\
\end{array}$ & 41 & 100,0 \\
\hline \multicolumn{3}{|l|}{ Busca informações sobre IST'S } \\
\hline $\operatorname{Sim}$ & 35 & 59,3 \\
\hline Não & 24 & 40,7 \\
\hline \multicolumn{3}{|l|}{ Se sim, onde busca? } \\
\hline Televisão & 08 & 22,9 \\
\hline Amigos & 06 & 17,1 \\
\hline Internet & 02 & 05,7 \\
\hline Profissional da saúde & 16 & 45,7 \\
\hline Todos & 03 & 08,6 \\
\hline Total & 35 & 100,0 \\
\hline \multicolumn{3}{|l|}{ Se considera vulnerável a adquirir uma IST } \\
\hline $\operatorname{Sim}$ & 08 & 13,6 \\
\hline Não & 48 & 81,4 \\
\hline Sem resposta & 03 & 05,1 \\
\hline \multicolumn{3}{|l|}{ Se sim, por quê? } \\
\hline Teve relação sexual desprotegida & 04 & 50,0 \\
\hline Não procura se informar sobre IST & 02 & 25,0 \\
\hline Tem parceiros (a) eventuais & 02 & 25,0 \\
\hline Tem vários parceiros (a) & 00 & 0 \\
\hline Total & 08 & 100,0 \\
\hline \multicolumn{3}{|l|}{ Se sente à vontade para falar sobre sexo } \\
\hline Sim & 35 & 59,3 \\
\hline Não & 24 & 40,7 \\
\hline \multicolumn{3}{|l|}{ Se não, por quê? } \\
\hline Tem vergonha & 8 & 33,3 \\
\hline Acha que sexo é para mais jovens & 2 & 08,3 \\
\hline Acha que idoso não tem relacão sexual & 10 & 41,7 \\
\hline Não gosta de falar sobre sexo & 4 & 16,7 \\
\hline Total & 35 & 100,0 \\
\hline Total & 59 & 100 \\
\hline
\end{tabular}

Fonte: Amaral SVA, et al., 2020. 


\section{DISCUSSÃO}

O gênero feminino representa a maior parte da amostra, o que se justifica, pela menor exposição das mulheres a riscos de acidentes ocupacionais, domésticos ou de trânsito, bem como de homicídio e suicídio. No Brasil as mulheres têm uma expectativa de vida de 79,4 anos, comparada a 72,2 anos entre os homens (IBGE, 2018; LEITE MT, et al., 2007). Além disso, a mulher possui mais acesso à informação relacionada à saúde, por culturalmente serem mais preocupadas com a saúde do que os homens, envolvendo-se mais com grupos da terceira idade (WICHMANN FMA, et al., 2013). A faixa etária predominante encontrada (70 e 79 anos) está em consonância com a expectativa de vida do brasileiro, que atualmente é de 75,8 anos (IBGE, 2018; SOUZA LPS, et al., 2013).

Verificou-se o uso prioritário dos serviços do Sistema Único de Saúde (SUS), em detrimento aos serviços privados, concordando com o Elsi-Brasil ao apontar que $75,3 \%$ dos idosos brasileiros dependem exclusivamente do SUS para o cuidado com sua saúde (MACINKO J, et al., 2018). Isto reforça a responsabilidade dos profissionais de saúde atuantes no SUS de acolher e atender os idosos em sua integralidade, fortalecendo vínculos e dirimindo barreiras, objetivando efetivar as ações de promoção da saúde e prevenção de doenças, entre elas as IST's.

O envelhecer é vivenciado de forma diferente entre as pessoas, alguns sofrem grande influência dos fatores culturais e morais em relação a seus comportamentos e condutas, contribuindo para submissão ao parceiro e isolamento social. Para outros, essa fase trata-se do início de outro ciclo, em que a diversão e a interação social devem ser experenciadas plenamente, inclusive a sexualidade, sendo essencial que seja vivida com responsabilidade e autonomia (LEITE MT, et al., 2007).

Quanto à escolaridade, evidenciou-se que a maioria possui poucos anos de estudo. Ressalta-se que o nível educacional dos idosos de hoje remete ao período da década de 20 a 50 , em que o acesso à educação ocorria de forma assimétrica de acordo com a classe social e gênero (CEZAR AK, et al., 2012). A escolaridade é relacionada como um determinante da situação de saúde/doença do idoso, e sua falta é considerada como um preceptor de doenças (SOUZA MDD, et al., 2016).

Daí a importância da assertividade das ações educativas, que precisam ser claras, e considerar o grau de compreensão das pessoas para alcançar o êxito esperado. Reconhecer as formas de transmissão e os aspectos relacionados às IST's é essencial em qualquer idade. Entretanto para idosos o significado é ainda maior, já que a falta de informação pode levar a prática de uma vida sexual insegura e resultar no adoecimento, que com a demora no diagnóstico e tratamento podem culminar em óbito em menor tempo, quando comparado a pessoas mais jovens (PAULINO MCFO, et al., 2014).

No que tange às condições financeiras a maioria informou uma renda de 1 a 5 salários mínimos. A renda tem um impacto importante na qualidade de vida, dignidade e autoestima, refletindo também na expectativa de vida (LEITE MT, et al., 2007). Idosos com menor recursos financeiros têm em geral, condições de saúde complicadas, dificuldade no acesso à informação e aos serviços de saúde, acarretando maior vulnerabilidade ao adoecimento (SOUZA MDD, et al., 2016).

O avanço da tecnologia, melhoria do acesso aos meios de comunicação e a evolução cultural na sociedade, desencadearam modificações nos comportamentos da população, que permitiu maior esclarecimento e conscientização acerca de informações importantes sobre a saúde e doença, dentre elas as IST'S (LEITE MT, et al., 2007). Isso justifica o conhecimento identificado entre os idosos de ao menos uma IST, igualmente constatado no estudo realizado em Montes Claros em que a maioria dos idosos independentemente do nível de escolaridade, relatou que conheciam mais de uma IST (SOUZA LPS, et al., 2013).

A divulgação, e maior acesso ao conhecimento são essenciais para a promoção à saúde, e prevenção de doenças. No entanto, é importante ressaltar que em relação ao conhecimento sobre das IST's, identificou-se um resultado relativamente satisfatório, mas muitos ainda apresentam um conhecimento deficiente, ao responder incorretamente ou não conseguir opinar. Um estudo realizado em Pernambuco revelou que os idosos tinham um conhecimento inferior sobre HIV/AIDS, quando comparado à adultos jovens com a mesma escolaridade (MELO HMA, et al., 2012). Esses dados demonstram que o conhecimento acerca das IST's ainda é mínimo, e necessita de ser aprimorado para que as ações de prevenção sejam mais assertivas. 
As estatísticas atuais indicam a senilização da infecção pelo HIV/AIDS o que pode ser explicado pela desconsideração por parte da sociedade, de que o idoso ainda tem vida sexual ativa, e por isso sujeito a riscos, seja por carência de informação e/ ou campanhas educativas pouco eficazes (NETO JD, et al., 2015; MELO HMA, et al., 2012; SANTOS AFM e ASSIS M, 2011, MS, 2017a).

Sabe-se que as IST's podem ser prevenidas por meio do uso de preservativo, bem como, em algumas delas, a vacinação. A literatura evidencia que a maioria das mulheres no período pós-menopausa, por não preocupar-se com anticoncepção, se veem pouco incentivadas a adesão ao preservativo, embora mantenham-se em alguns casos sexualmente ativas, expondo-se, portanto, ao risco de contrair uma IST (LAROQUE MF, et al., 2011). No universo desse estudo os idosos demonstraram conhecimento sobre a importância do uso do preservativo, e não o associaram somente a ideia da anticoncepção, revelando a consciência do caráter preventivo. Infere-se que a participação em um grupo de convivência seja um fator importante para se ter maior acesso à informação, e influência neste comportamento.

O comportamento dos idosos desta pesquisa assemelha-se ao identificado na Paraíba pois a maioria dos participantes, também conheciam as formas de transmissão das IST's (BRITO NM, et al., 2016). Sabe-se que a transmissão via sexual é responsável por $75 \%$ a $85 \%$ de todos os casos de HIV/ AIDS. O que fundamenta a discussão das práticas de comportamento sexual seguro entre os parceiros para a prevenção da infecção não só do HIVIAIDS, assim como das demais IST's (SOUZA MDD, et al., 2016).

Entretanto embora esbocem este conhecimento, a maioria dos idosos pesquisados que se encontram ativos sexualmente mostram-se resistentes ao uso do preservativo, justificando tal fato por terem parceiro fixo. Em Catanduva-SP, 80\% dos idosos entrevistados admitiram não usar preservativo (BURIGO GF, et al., 2015). O mesmo foi constatado por Paulino MCFO, et al., (2014) e Laroque MF, et al., (2011), ao identificar que este público conhece a importância do preservativo para prevenção das IST's, porém são resistentes ao uso.

Essa baixa adesão ao uso do preservativo pelos idosos relaciona-se ao pensamento distorcido de invulnerabilidade, atrelado a fatores socioculturais e concepções pré-fixadas, de que indivíduos da terceira idade com parceiro fixo não são susceptíveis às IST's (LAROQUE MF, et al., 2011; SOUZA MDD, et al., 2016; MOREIRA TM, et al., 2012). Estudos demonstram que quanto maior a confiança, menor a percepção de vulnerabilidade, consequentemente maior é o risco as diversas IST'S (LEITE MT, et al., 2007; PAZ MA, et al., 2013). Ressalta-se que o uso de preservativo é um comportamento incorporado a partir da década de 80 , e, portanto, não fazia parte da juventude dos idosos, o que pode justificar o não reconhecimento deste como uma fonte de prevenção das IST's (LAROQUE MF, et al., 2011; SOUZA MDD, et al., 2016).

A discussão sobre o uso do preservativo durante relações consideradas "seguras" e "estáveis" é complexa e ainda cercada de preconceitos, mitos e tabus, porém necessária visto que o estado civil não é um indicador confiável para avaliação do comportamento sexual e dos riscos existentes. Portanto constitui-se uma temática relevante para ser levantada nos espaços de educação em saúde (SOUZA LPS, et al., 2012).

Outro resultado importante é a constatação da ciência por parte deles da vacina contra a hepatite B, identificando-a como uma doença imunoprevenível, disponível inclusive para sua faixa etária. Porém no Brasil os dados ainda são alarmantes, pois os casos confirmados de hepatite B entre indivíduos maiores de 60 anos, passou de 448 (2006) para 1129 (2016) (MS, 2017b).

Como constatado os idosos evitam falar sobre o assunto, seja por vergonha ou por acharem que não deveriam mais manter-se ativos. Soma-se a isto os estereótipos presentes até entre os profissionais de saúde, que por uma visão deturpada acerca do envelhecimento, os enxerga como doentes, dependentes, incapazes e poliqueixosos (SOUZA L e RIBEIRO AP, 2013).

Entretanto, envelhecer não é tornar-se assexuado, e estes fatores socioculturais dificultam o exercício de uma vida sexual integral e segura (UCHÔA YS, et al., 2016). Visualiza-se, portanto, a iminente necessidade de alteração dessa cultura na qual os profissionais de saúde, dentre eles os enfermeiros, passem a considerar a sexualidade na terceira idade. Tornando crucial a discussão dessa temática em ações educativas, de forma a sensibilizá-los para a mudança de atitude e de comportamento, visando a promoção de um envelhecimento ativo, saudável e seguro (SOUZA L e RIBEIRO AP, 2013; PAZ MA, et al., 2013). 


\section{CONCLUSÃO}

O estudo evidenciou que a maioria dos idosos possuem conhecimentos limitados sobre às IST's, seus métodos de prevenção, e uma baixa adesão ao uso preservativo nas relações sexuais. Achados preocupantes, e que alertam para a necessidade da equipe de saúde, melhorar suas ações voltadas para esse público. Dentre as medidas primordiais destacam-se as ações educativas, o que permitirá ampliar o conhecimento geral dos idosos sobre essas doenças, suas formas de prevenção, bem como conscientizá-los do seu grau de vulnerabilidade que ainda é tão ignorada. Além disso, reforçar-se a necessidade de se elaborar demais estratégias para um diagnóstico cada vez mais precoce e com tratamento adequado das IST's neste público. Por fim, ressalta-se que a amostra por conveniência pode ser elencada como uma limitação desse estudo, e por isso orienta-se cautela na generalização dos dados apresentados, por não se tratar de investigação por base populacional, embora houvesse o cuidado de realizar a coleta de dados com a inclusão do maior número possível de participantes.

\section{REFERÊNCIAS}

1. ANDRADE JAA, et al. Vulnerabilidade de idosos a Infecções sexualmente transmissíveis. Acta Paulista Enfermagem, 2017; 30(1): 8-15.

2. BRITO NM, et al. Idosos, infecções sexualmente transmissíveis e aids: conhecimentos e percepção de risco. ABCS Health Sciences, 2016; 41(3), 140-145.

3. BURIGO GF, et al. Sexualidade e comportamento de idosos vulneráveis a doenças sexualmente transmissíveis. Revista CuidArte Enfermagem, 2015; 9 (2), 148-153.

4. CEZAR AK, et al. Prevenção de doenças sexualmente transmissíveis na visão de idosos de uma estratégia da saúde da família. Revista Brasileira de Enfermagem, 2012; 65(5), 745-50.

5. GIL AC. Como elaborar projetos de pesquisa. São Paulo: Atlas. 2010

6. INSTITUTO BRASILEIRO DE GEOGRAFIA E ESTATÍSTICA. IBGE: Estatísticas Sociais [online], 2018.

7. LAROQUE MF, et al. Sexualidade do idoso: comportamento para a prevenção de DST/AIDS. Revista Gaúcha Enfermagem, 2011; 32(4), 774-780.

8. LEITE MT, et al. Doenças sexualmente transmissíveis e HIV/AIDS na opinião de idosos que participam de grupos de terceira idade. Revista Brasileira de Geriatria e Gerontologia, 2007; 10 (3), 339-354.

9. MACINKO J, et al. Primary care and healthcare utilization among older Brazilians (ELSI-Brasil). Rev Saude Publica. 2018;52, Supl 2:6s.

10. MASCHIO MBM, et al. Sexualidade na terceira idade: medidas de prevenção para doenças sexualmente transmissíveis e AIDS. Revista Gaúcha Enfermagem, 2011: 32(3), 583-9.

11. MELO HMA, et al. O conhecimento sobre Aids de homens idosos e adultos jovens: um estudo sobre a percepção desta doença. Ciência e Saúde Coletiva, 2012; 17(1), 43-53.

12. MIRANDA GMD, et al. O envelhecimento populacional brasileiro: desafios e consequências sociais atuais e futuras. Revista Brasileira de Geriatria e Gerontologia, 2016; 19(1), 507-519.

13. Ministério da Saúde (BR). Conselho Nacional de Saúde. Resolução no 510, de 07 de abril de 2016: Dispõe sobre as normas aplicáveis a pesquisas em Ciências Humanas e Sociais. Brasília (DF): MS; 2016

14. Ministério da Saúde (BR). Departamento de Vigilância, Prevenção, Controle das IST, do HIV/AIDS e das Hepatites Virais. BolEpidemiol HIV/AIDS. Brasília (DF): MS; 2017a.

15. Ministério da Saúde (BR). Departamento de Vigilância, Prevenção, Controle das IST, do HIV/AIDS e das Hepatites Virais. BolEpidemiol Hepatites virais. Brasília (DF): MS; 2017b.

16. Ministério da Saúde (BR). Departamento de Vigilância, Prevenção, Controle das IST, do HIV/AIDS e das Hepatites Virais. Virais. BolEpidemiol Sífilis. Brasília (DF): MS; 2017c.

17. Ministério da Saúde (BR). Secretaria de Vigilância em Saúde. Departamento de Vigilância de Doenças Transmissíveis. Coordenação- Geral do Programa Nacional de Imunizações. Informe Técnico sobre a vacina Papilomavírus Humano (HPV) na Atenção Básica. Brasília (DF): MS; 2014.

18. MOREIRA TM, et al. Conhecimento das mulheres idosas sobre doenças sexualmente transmissíveis, conhecimento, uso e acesso aos métodos preventivos. Revista Eletrônica de Enfermagem, 2012; 14 (4), 803-10.

19. NETO JD, et al. Doenças Sexualmente Transmissíveis em idosos: uma revisão sistemática. Revista Ciência e Saúde Coletiva, 2015; 20 (12).

20. OLIVI M, et al. Behavior, knowledge and perception of risks about sexually transmitted diseases in a group of people over 50 years old. Revista Latino Americana Enfermagem, 2008; 16(4), 679-685.

21. PAZ MA, et al. The influence of the usage of the male condon by seniors in the vulnerability to HIV: a systematic review with meta-analysis. J Bras Doencas Sex Transm, 2013; 25 (3), 150-6.

22. PAULINO MCFO, et al. Análise dos comportamentos sexuais de idosos cadastrados em uma estratégia saúde da família, Revista Kairós Gerontologia, 2014; 17(4), 49-61.

23. SANTOS AFM e ASSIS M. Vulnerabilidade das idosas ao HIV/AIDS: despertar das políticas públicas e profissionais de saúde no contexto da atenção integral: revisão de literatura. Rev Bras Geriatr Gerontol, 2011 ; 16 (4), 821 -832. 
24. SOUZA LPS, et al. Análise da clientela idosa portadora de HIV atendida em um centro ambulatorial em Montes Claros. Rev Bras Geriatr Gerontol, 2012; 15(4), 767-776.

25. SOUZA LPS, et al. Sexualidade na terceira idade: conhecimento e comportamento de idosos residentes em um município de Minas Gerais. Enfermagem em Foco, 2013; 4 (3-4), 187-190.

26. SOUZA L, e RIBEIRO AP. Prestar cuidados de enfermagem a pessoas idosas: experiências e impactos. Saúde Soc. São Paulo. 2013; 22 (3), 866-877.

27. SOUZA MDD, et al. Conhecimento dos idosos da estratégia saúde da família em relação ao HIV/AIDS. Rev enferm UFPE, 2016; 10 (11), 4036-45.

28. TOLDRÁ RC, et al. Promoção da saúde e da qualidade de vida com idosos por meio de práticas corporais. Revista O Mundo da Saúde,2014; 38 (2), 159-168.

29. UCHÔA YS, et al. A sexualidade sob o olhar da pessoa idosa. Rev Bras Geriatr Gerontol, $2016 ; 19$ (6), $939-949$.

30. WICHMANN FMA, et al. Grupos de convivência como suporte ao idoso na melhoria da saúde. Rev Bras Geriatr Gerontol, 2013; 16(4), 821-832. 